El ect ron Cycl ot ron emi ssi on di agnost i cs for hel i cal pl asma in the I arge hel i cal devi ce

\begin{tabular}{|l|l|}
\hline $\begin{array}{l}\text { j our nal or } \\
\text { publ i cat i on } \mathrm{titl} \text { e }\end{array}$ & I EEE Tr ansact i ons on PI asma Sci ence \\
\hline vol une & Vol . 32 \\
\hline number & I ssue 4| Part 3 \\
\hline page r ange & pp. 1716- 1720 \\
\hline year & 2004 08 01 \\
\hline URL & ht t p: //hdl . handl e. net /10655/3794 \\
\hline
\end{tabular}




\title{
Electron Cyclotron Emission Diagnostics for Helical Plasma in the Large Helical Device
}

\author{
Yoshio Nagayama, K. Kawahata, Shigeru Inagaki, Tomohiro Morisaki, and K. Narihara
}

Abstract-Electron cyclotron emission (ECE) diagnostics have been developed in the large helical device (LHD). The ECE is transmitted over $80 \mathrm{~m}$ from the antenna to the spectrometer with the corrugated waveguide system, which has a low transmission loss $(\sim 30 \%)$. The polarization of ECE is identical for all frequencies, while the field angle varies from $-40^{\circ}$ to $+30^{\circ}$ on the ECE sight line. The ECE temperature measured by the Michelson, which is calibrated with a hot source, agrees well with Thomson scattering measurement in a wide range of the electron density. Magnetic island formation is investigated using the electron temperature profile measured with the Michelson. The radiometer is cross-calibrated to the Michelson and is employed for the study of fast phenomena, such as the sa wtooth oscillation. Besides the normal ECE, tangential ECE is investigated in LHD in order to explore the potential of ECE diagnostics.

Index Terms-Diagnostics, electron cyclotron emission (ECE), large helical device (LHD), microwave, plasma, temperature.

\section{INTRODUCTION}

$\mathbf{T}$ THE electron cyclotron emission (ECE) measurement is one of the useful diagnostics for the local electron temperature [1], [2], as the frequency is proportional to the magnetic field, which is different at different positions. In tokamaks, the ECE diagnostics are employed as a powerful tool to study magnetohydrody namic (MHD) instabilities [3]. This is because ECE has the capability of continuous measurement with high time resolution, as the intensity is proportional to the electron temperature if ECE is the black body radiation, which is usually a good approxi mation in high-temperature and high-density plasmas.

Recently, helical systems have been recognized as strong alternative concepts because of their potential for a steady-state fusion reactor. The world's largest helical device is the large helical device (LHD), which has a major radius of $R_{\mathrm{ax}}=3.5-3.9 \mathrm{~m}$, an averaged minor radius of $a_{\mathrm{p}}=0.6-0.65 \mathrm{~m}$, and a magnetic field of $B_{\mathrm{ax}}=2.6-2.9 \mathrm{~T}$ [4]. The major differences between LHD and a tokamak are as follows: 1) the magnetic field profile is peaked in LHD, while it is monotonic in tokamaks in terms of the major radius $\mathrm{R}$ and 2) the angle of the magnetic field changes from $-40^{\circ}$ to $+30^{\circ}$ in LHD, while it is almost constant in tokamaks.

In tokamaks, the radial profile of the electron temperature can be obtained by means of the ECE measurement from the

Manuscript received August 27, 2003; revised November 28, 2003. This work was supported in part by the Ministry of Education, Science, Sports and Culture under Grant-in-Aid for Scientific Research (B) 11480116 and Grant-in-Aid for Scientific Research (B) 14208055.

The authors are with the Large Helical Device Group, National Institute for Fusion Science, Toki 509-5292, Japan (e-mail: nagayama@ nifs.ac.jp).

Digital Object Identifier 10.1 109/TPS.2004.833380

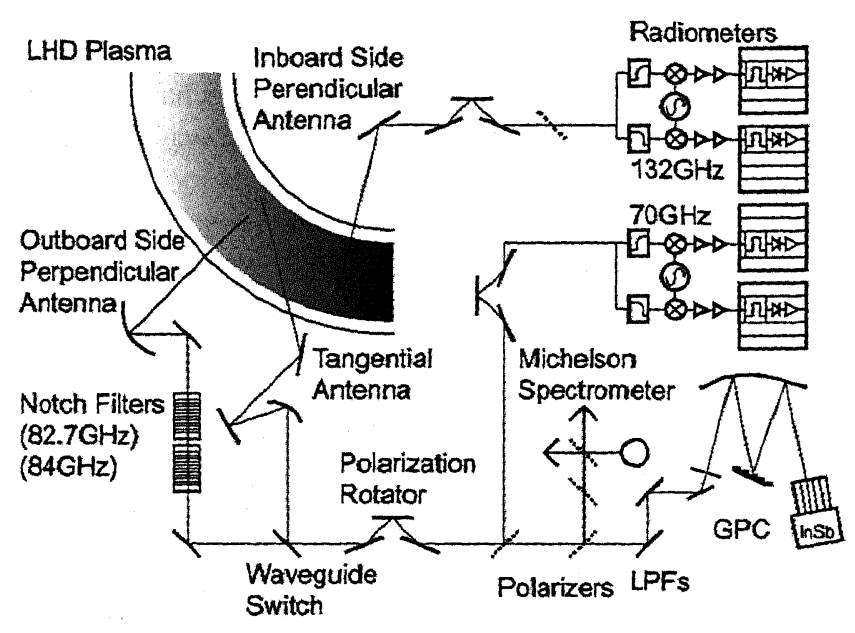

Fig. 1. Schematic diagram of the ECE measurement system in LHD.

outboard side since the magnetic field profile is monotonic. In tokamaks, however, the edge electron temperature at the inboard side cannot be obtained due to the third harmonics of the ECE from the outboard side. In the LHD configuration, only half of the plasma region (i.e., axis to outer edge or axis to inner edge) can be measured. The full radial profile can be measured without the disturbance of the third harmonics by using two antennas.

A number of different ECE systems have been developed to diagnose the plasma in LHD [5]-[12]. This paper will present the overview of the ECE diagnostics in LHD. In Section II, the LHD configuration and the ECE measurement system will be presented. In Section III, the polarization will be presented. In Section IV, the electron temperature measurement using ECE will be presented. In Section V, the time evolution of the magnetic island and the sawtooth oscillation will be presented as examples of physics research using the ECE diagnostics in LHD.

\section{ECE MEASUREMENT SYSTEM}

Fig. 1 shows a schematic diagram of the ECE measurement system in LHD. In LHD, two perpendicular antennas, one at the inboard and one at the outboard side, respectively, are installed to measure the full electron temperature profile. An additional tangential antenna is installed to give additional information about the central temperature at low densities. The ECE is transferred from the antenna in the LHD hall to the spectrometers in the diagnostics room using the corrugated waveguide. The length of the transmission line is $\sim 80 \mathrm{~m}$. The measured transmission loss is $\sim 30 \%$ between $80-220 \mathrm{GHz}$. The waveguide has an inner wall with a diameter of $63.5 \mathrm{~mm}$ and with rectangular grooves with a depth of $0.43 \mathrm{~mm}$, a width of $0.46 \mathrm{~mm}$, and a 
(a)

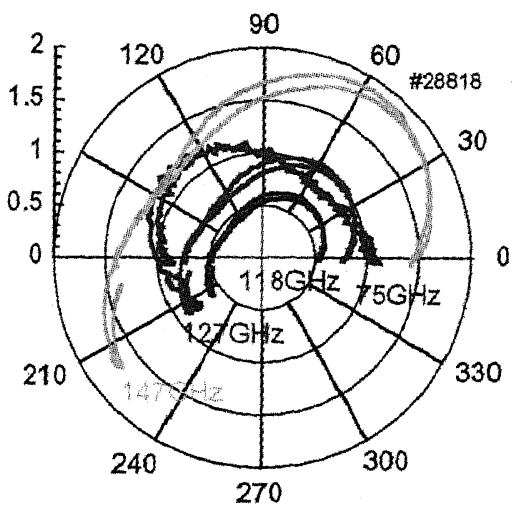

(b)

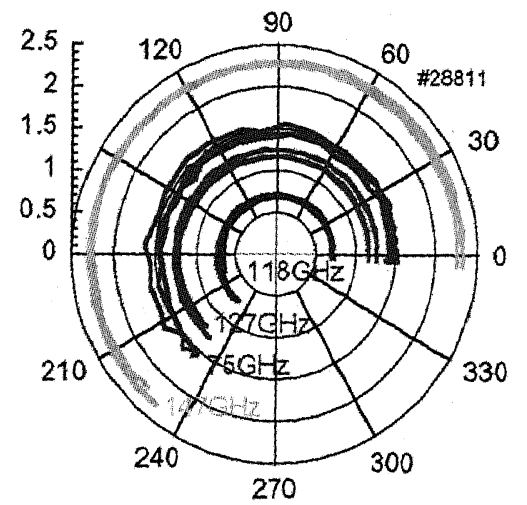

Fig. 2. (a) Polarization of the ECE received by the perpendicular antenna. $75-\mathrm{GHz}$ signal is $\mathrm{O}$ mode, whereas the other ones are $\mathrm{X}$ mode. (b) Polarization of the ECE received by the tangential antenna.

pitch of $0.66 \mathrm{~mm}$. It is designed to transfer the microwave with frequencies between $50-220 \mathrm{GHz}$. The $\mathrm{HE}_{11}$ mode is selected and has a high transmittance in the corrugated waveguide, and the $\mathrm{HE}_{11}$ mode at the end of the waveguide couples well to the Gaussian beam in free space. The beam waist of the Gaussian beam is $\sim 60 \%$ of the diameter of the corrugated waveguide. The antenna is designed so the $100-\mathrm{GHz}$ microwave has the beam waist of $38 \mathrm{~mm}$ at the inlet of the waveguide and that of $80 \mathrm{~mm}$ at the $R=3.95 \mathrm{~m}$ in the plasma.

An $84-\mathrm{GHz}$ notch filter and an $82.6-\mathrm{GHz}$ notch filter are installed behind a short waveguide at the front end of the corrugated waveguide in order to reduce the leaked $\mathrm{ECH}$ microwaves [5]. Each notch filter is an interference filter made of 200 sheets of $25-\mu \mathrm{m}$-thick polypropylene film. The transmission loss is more than $30 \mathrm{~dB}$ and the full width of the transmission loss of each filter is $\sim 1 \mathrm{GHz}$. Notch filters in the rectangular waveguide in front of the radiometer attenuate by $40 \mathrm{~dB}$ at $82.6 \mathrm{GHz}$ and $84 \mathrm{GHz}$ with a full width of $\sim 0.5 \mathrm{GHz}$.

The fundamental O-mode ECE received by the outboard antenna is separated by a wire-grid beam splitter and is measured with a low frequency radiometer. The second harmonic $\mathrm{X}$-mode ECE received by the antenna in the outboard side is measured with the fast scanning Michelson interferometer (Michelson) and with a grating polychromator (GPC) [9], [10]. In the Michelson, a roof-top mirror in the interferometer oscillates with the active scan length of $4.6 \mathrm{~cm}$ and with a scanning frequency of $20 \mathrm{~Hz}$. Each scan produces an interferogram, which is to be Fourier transformed to produce a spectrum of the ECE signal from $\sim 60$ to $300 \mathrm{GHz}$. The GPC has a Czerny-Turner type setup and the frequency range between 109 and $176 \mathrm{GHz}$ is detected with the InSb detector array of 14 channels.

The second harmonic X-mode ECE received by the inboard antenna is measured with a high-frequency heterodyne radiometer. In the radiometer, the ECE with frequencies higher and lower than the local oscillator are separated by band-pass filters [11]. Each separated ECE is mixed with the local frequency. The intermediate frequency is amplified by $25 \mathrm{~dB}$ and is separated into another two frequency bands. Each frequency band is amplified by $40-50 \mathrm{~dB}$ and is divided to eight channels by an eight-way power divider. Each channel consists of a band-pass filter with the bandwidth of $1.2 \mathrm{GHz}$, a diode, and a dc amplifier. So, the ECE is divided to 32 channels. The high-frequency radiometer has a local oscillator at $132 \mathrm{GHz}$ and covers the frequency range between 106 and $158 \mathrm{GHz}$. The low-frequency radiometer has a local oscillator at $70 \mathrm{GHz}$ and covers the frequency range between 53 and $87 \mathrm{GHz}$. Data of the GPC and radiometers are separated to slow and fast CAMAC digitizers with 12-bit ADC. The sampling rates of the slow and fast digitizers are 10 and $200 \mathrm{kHz}$, respectively.

Since two ECE systems are required to observe the full radial profile of $T_{e}$ in the LHD configuration, two fast sampling ECE systems, such as the GPC or the radiometer, are installed. The frequency range of the GPC can be changed, and the sensitivity of the radiometer is high. The Michelson is useful to observe the full ECE spectrum, and its sensitivity can be absolutely calibrated.

\section{NORMAL AND TANGENTIAL ECE}

As is described in Section I, the angle $\theta$ of the magnetic field vary from $-40^{\circ}$ to $+30^{\circ}$ in LHD. The ECE propagation is calculated numerically, with the assumption that the electron temperature profile is parabolic and the electron density profile is flat [6]. Theoretical result is as follows: the ECE polarization is determined at the plasma edge, if the electron density sharply drops to zero at the plasma edge. Usually, these are good assumptions in LHD. A universal polarization rotator is set in each waveguide system. It consists of a standard $90^{\circ}$ miter bend between two $135^{\circ}$ miter bends. This device rotates the polarization of the linearly polarized incident radiation by two times the angle between the planes of these miter bends and the incident electric field. During long pulse operation, a pulse motor turns the universal polarization rotator.

Fig. 2(a) shows the polarization of the ECE of different frequencies received by the perpendicular antenna. The $75-\mathrm{GHz}$ signal is $O$ mode, whereas the other ones are $X$ mode. While the field angle changes spatially, the second harmonic ECE in a wide frequency range has identical polarization as expected in the above discussion. The fundamental ECE also has a single polarization, which is $90^{\circ}$ different from the second harmonics. Therefore, the fundamental ECE is mainly $\mathrm{O}$ mode and the 

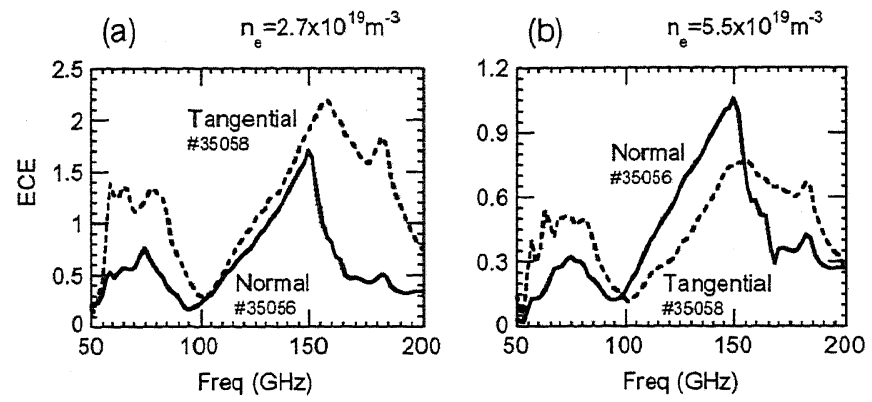

Fig. 3. Comparison of the ECE spectra received by the tangential and the perpendicular antennas in case of a low (a) and a high (b) density plasma.

second harmonic is X mode. Fig. 2(b) shows that the ECE received by the tangential antenna is not linearly polarized.

Fig. 3 shows the spectrum of the ECE received by the normal antenna and the tangential one. The normal ECE spectrum is cut off over $150 \mathrm{GHz}$, which corresponds to the cutoff of the ECE from the core region. ECE from the plasma center may be detected in the tangential view, since the $|B|$ profile in this case is monotonic. In the case of low density [Fig. 3(a)], the tangential ECE is similar to the normal one, and the ECE from the plasma center $(160 \mathrm{GHz})$ is peak. In the case of high density [Fig. 3(b)], tangential ECE is low, since the ECE ray is refracted. In a tokamak, the tangential ECE is used to observe the effect of nonthermal electrons [13]. In LHD, the effect of nonthermal emission is not clear.

\section{ELECTRON TEMPERATURE}

\section{A. Calibration}

The ECE antenna at the outboard side consists of a concave mirror and a convex mirror. In the case of calibration, the concave mirror rotates $180^{\circ}$ to look at a hot $(873 \mathrm{~K})$ radiation source. Also doing calibration, a polarizer that is set at $45^{\circ}$ in the plasma experiment, is set at $90^{\circ}$ in order to increase the calibration signal, and an active low-pass filter is additionally used in order to reduce the bit noise. The active low-pass filter has a gain of 200 and it cuts the frequencies higher than $100 \mathrm{kHz}$. The scanning frequency of the Michelson is $5 \mathrm{~Hz}$ in the case of calibration, since it works almost continuously. The one million scans of the interferogram signal of the Michelson are integrated for the calibration. The interferogram of the room temperature radiation subtracts that of the hot/cold source. Taking the Fourier transform of the subtracted interferogram gives the frequency spectrum of the hot/cold radiation.

The hot source is a plane ceramic heater with an area of $25 \mathrm{~cm}^{2}$. The cold source is a liquid nitrogen cooled foam absorber. The hot/cold ratio is defined as

$$
\text { Hot } / \text { Cold }=\frac{I_{\text {hot }}}{I_{\text {cold }}} \frac{\left(T_{\text {room }}-T_{\text {cold }}\right)}{\left(T_{\text {hot }}-T_{\text {room }}\right)}
$$

where $I_{\text {hot }}$ and $I_{\text {cold }}$ are the spectrum of hot and cold source subtracted the room temperature radiation, respectively, and $T_{\text {room }}, T_{\text {cold }}$, and $T_{\text {hot }}$ are the temperature of the room, the cold source, and the hot source, respectively. The emissivity of the hot source is $\sim 90 \%$. The calibration coefficient $\mathrm{Calb}$ is determined from: the spectrum of the radiation of the hot

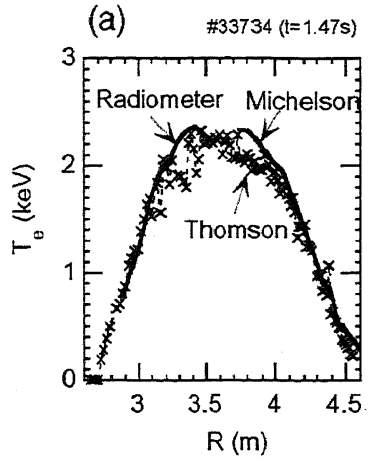

(b)

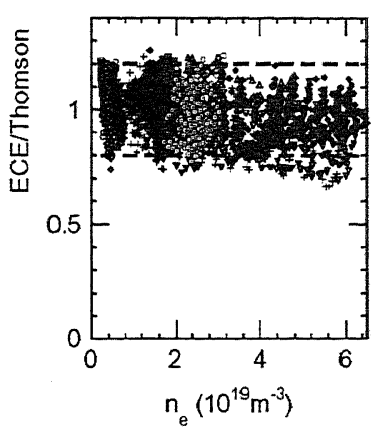

Fig. 4. (a) Electron temperature profile measured by the Thomson scattering (x) and the ECE diagnostics in the case of lower beta. (b) Ratio of the ECE and Thomson scattering for the various line-averaged electron density. Gray line indicates $T_{\mathrm{ECE}}=T_{\text {Thomson }}$. Broken lines indicate the discrepancy of $\pm 20 \%$. Different symbols indicate different shots.

source subtracted by that from the room temperature $I_{\text {antenna }}$ and the gain of the active filter Amp as

$$
\text { Calb }=\frac{\left(T_{\text {hot }}-T_{\text {room }}\right)}{I_{\text {antenna }}} \cdot \operatorname{Amp} \cdot \frac{\text { Hot } / \text { Cold }}{45^{\circ} / 90^{\circ}} .
$$

\section{B. Electron Temperature Profile}

Fig. 4(a) shows the electron temperature profile measured using ECE and the Thomson scattering when $B_{\mathrm{ax}}=2.8 \mathrm{~T}$ and $R_{\mathrm{ax}}=3.6 \mathrm{~m}$. The radiometer data are cross calibrated to the Michelson under the assumption that the electron temperature is identical at the same $\rho$. Here, $\rho$ is the normalized minor radius. In Fig. 4 (b), the ratio of the electron temperature at $\rho=0.2$ measured by the ECE to that by the Thomson scattering is plotted versus the line averaged electron density. The electron temperature measured by the ECE and the Thomson agrees within $\pm 20 \%$ for a wide range of density.

In the case of higher density, the ECE temperature looks slightly less than the Thomson temperature. This can be explained as follows: the ECE ray is diffused due to the twisted plasma structure in LHD, when the electron density is close to the cutoff density $n_{c}$ of the $\mathrm{X}$ mode. Actually, it has been observed that ECE temperature starts to decrease linearly as the density increases when $n_{e}>0.6 n_{c}$ in LHD [8]. In this case, since the cutoff density is $n_{c} \sim 10^{20} \mathrm{~m}^{-3}$, the ECE diagnostics should be affected when $n_{e}>6 \times 10^{19} \mathrm{~m}^{-3}$.

\section{STUDY OF ISLAND AND SAWTOOTH OSCILLATION}

\section{A. Magnetic Island Formation}

The magnetic island structure in hot magnetized plasmas has been an important research topic for many decades [14], [15]. It has been considered that the magnetic island, which is generated due to the error field, significantly degrades plasma confinement in a helical system. Recently, however, it has been observed that the magnetic island structure produced by the error field is diminished in plasma [16], [17]. LHD is one of the most suitable devices to study the physics of the magnetic island, since it can be generated and controlled by adding the $(m, n)=(1,1)$ error field, which is generated by the local island diverter (LID) coil current. The electron temperature profile measured with ECE is 
(a)
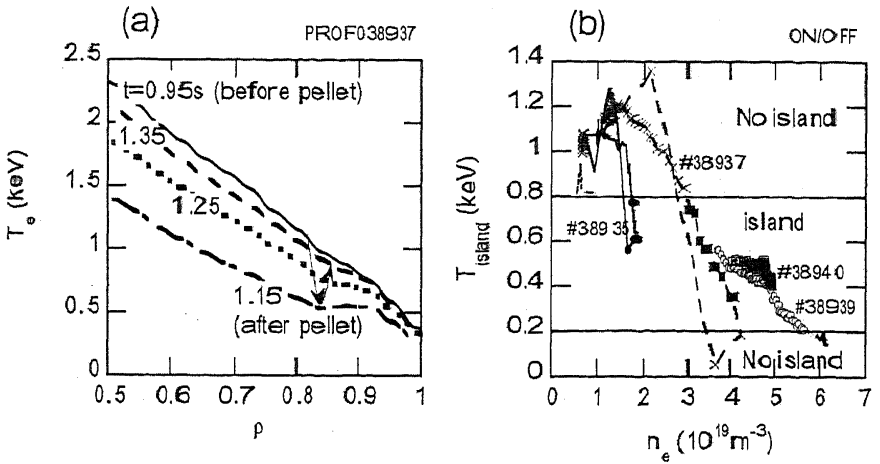

Fig. 5. (a) Time evolution of electron temperature profiles of LHD plasmas by the ECE (Michelson) before $(t=0.95 \mathrm{~s})$ and after the hydrogen ice pellet injection $(\mathrm{t}=1 \mathrm{~s})$. Here, $R_{\mathrm{ax}}=3.6 \mathrm{~m}, B_{\mathrm{ax}}=2.8 \mathrm{~T}$, and $I_{\mathrm{LID}}=-1200 \mathrm{~A}$, which makes the $(m, n)=(1,1)$ error field of $7 \times 10^{-4} \mathrm{~T}\left(\mathrm{I}_{\mathrm{LI}}=-1200\right.$ A). (b) Trajectories of the electron temperature at the island versus the electron density during a single discharge. Island structure is observed at the region indicated by circular or rectangular marks. Here, $R_{a x}=3.6 \mathrm{~m}$ and $B_{a x}=$ $2.8 \mathrm{~T}$.

use ful to observe the magnetic island structure, since the noise is much smaller than the signal.

Fig. 5(a) shows the time evolution of $T_{e}$ profiles of the LHD plasmas with the LID field before and after the hydrogen ice pelletinjection. The LID current $\left(I_{\mathrm{LID}}\right)$ is $-1200 \mathrm{~A}$, which provides the $(1,1)$ error field of $7 \times 10^{-4} \mathrm{~T}$ at $R=3.9 \mathrm{~m}$. The island region is flat in the temperature profile. A remarkable observation is as follows: The island structure is not observed before the ice pellet injection, while the LID current is large enough to produce the vacuum magneticisland structure. The island structure appears after the ice pellet injection. The island structure shrinks as the electron temperature increases.

The magnetic island may not depend on the electron density. Fig. 5(b) shows trajectories of the electron temperature at the island versus the electron density during a single discharge. This trajectory indicates that the island structure is observed in the limited region $\left(0.2 \mathrm{keV}<T_{e}<0.8 \mathrm{keV}\right)$ of the electron temperature at the island in any density range. The magnetic island does not appear in the plasma when the electron temperature $\left(T_{e}\right)$ is too high or too low in LHD. These are observed in various plasmas with different conditions.

\section{B. Sawtooth Oscillation}

The sawtooth oscillation is a series of periodic crashes. In LHD, the kink, the tearing, and the ballooning modes should be stable, since the plasma current is very low and the shear is high. Nevertheless, sawtooth oscillations have been observed in LHD [18]. Fig. 6 shows the time evolution of the electron temperature $\left(T_{\mathrm{e}}\right)$ showing the sawtooth oscillation in the helical plasma with the toroidal current in the counter direction. The $T_{e}$ profile is measured with the high-frequency radiometer, and the radiometer data is cross calibrated to the Michelson interferometer. The plasma has $B_{\mathrm{ax}}=2.8 \mathrm{~T}$ and $R_{\mathrm{ax}}=3.6 \mathrm{~m}$, and it is heated by the counterbeam with the deposited power of 6.2 MW. The plasma current is driven by the tangential neutral beaminjection (NBI) in the counter direction.

Interestingly, no precursor oscillation has been observed in the ECE signals. As the crash time of $\sim 1 \mathrm{~ms}$ is much shorter than the period of plasma rotation, we cannot know whether a

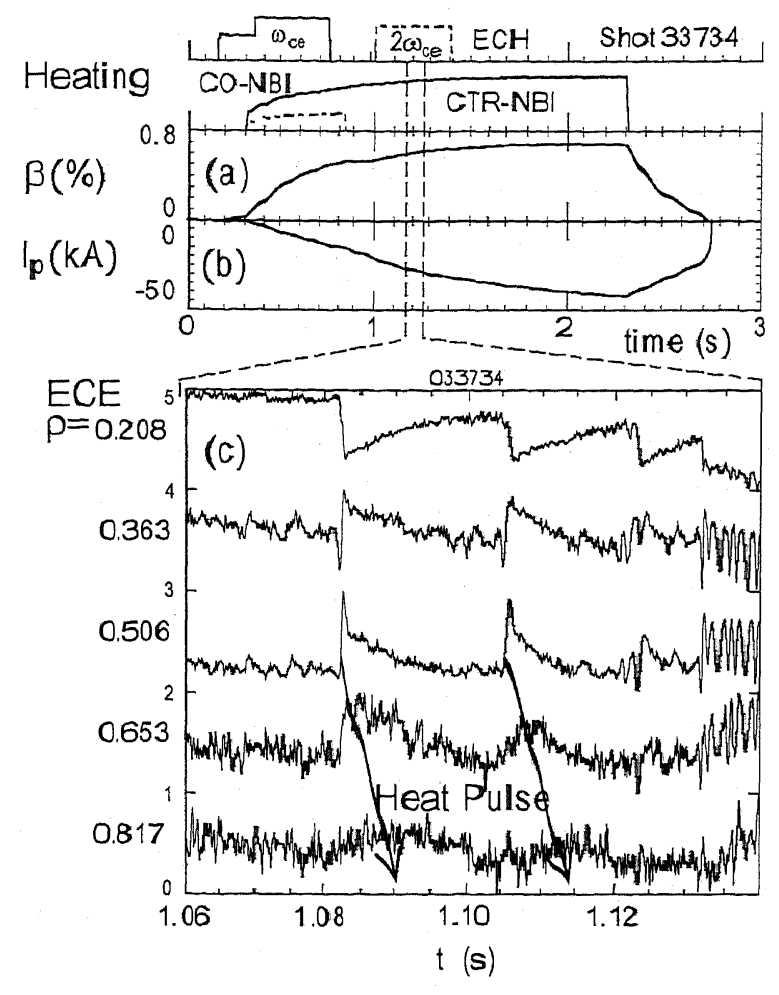

Fig. 6. Heating and plasma parameters in a counter-NBI heated LHD plasma with sawtooth oscillation: (a) plasma $\beta$, (b) plasma current $\left(\boldsymbol{I}_{\mathrm{p}}\right)$, and (c) ECE signals.

precursor does exist, or not. Outward heat pulse propagation in the case of a full crash has been observed. Observed rapid mixture of the plasma in the limited region suggests that sawtooth crashes in LHD are reconnection phenomena. The localized mode may cause the crash, but in the present theory the ballooning mode is usually stable due to the reversed shear in LHD. The driving instability of the sawtooth crash has not been identified in LHD. Further theoretical and experimental works are needed to understand the sawtooth oscillation in a helical system.

\section{CONCLUSION}

For ECE diagnostics, the $|B|$ profile, field angle, and twisted plasma structure in a helical system are much different from a tokamak. This study demonstrates that, like in tokamaks, the electron temperature in LHD can be measured by means of ECE, since thepolarization is well aligned in the different frequencies. In LHD, ECE diagnostics are utilized as a pow erful tool to study the island formation since the relative error of the $T_{e}$ profile is so small and to study the saw tooth oscillation and the heat pulse propagation since time and spatial resolutions of ECE are so good.

\section{REFERENCES}

[1] A. E. Costley, R. J. Hastie, J. W. M. Paul, and J. Chamberlain, "Electron cyclotron emission from a tokamak plasma: Experiment and theory," Phys. Rev. Lett., vol. 33, no. 13, pp. 758-761, Sept. 1974.

[2] M. Bornatici, R. Cano, O. De Barbieri, and F. Engelmann, "Electron cyclotron emission and absorption in fusion plasmas," Nucl_ Fusion, vol. 23, no. 9, pp. 1153-1257, S ept. 1983. 
[3] Y. Nagayama, M. Yamada, W. Park, E. D. Fredrickson, A. C. Janos, K. M. McGuire, and G. Taylor, "Tomography of full sawtooth crashes on the tokamak fusion test reactor," Phys. Plasmas, vol. 3, no. 5, pp. 1647-1655, May 1996.

[4] O. Motojima et al., "Initial physics achievements of large helical device experiments," Phys. Plasmas, vol. 6, no. 5, pp. 1843-1850, May 1999.

[5] Y. Nagayama et al., "Electron cyclotron emission diagnostics on the large helical device," Rev. Sci. Instrum., vol. 70, no. 1, pp. 1021-1024, Jan. 1999.

[6] P. C. de Vries et al., "The polarization of electron cyclotron emission spectra in the large helical device," Phys. Plasmas, vol. 7, no. 9, pp. $3707-3713$, Sept. 2000.

[7] Y. Nagayama et al., "Overview of ECE diagnostics on LHD, fusion engineering and design," Fusion Eng. Design, no. 1-4, pp. 201-211, Jan. 2001 .

[8] H. Sasao et al., "ECE diagnostics in the high density plasma in LHD," Fusion Eng. Design, vol. 53, no. 1-4, pp. 153-160, Jan. 2001.

[9] P. C. de Vries, K. Kawahata, Y. Nagayama, S. Inagaki, H. Sasao, and Y. Ito, "Electron cyclotron emission measurements by means of a grating polychromator on the large helical device," Fusion Eng. Design, vol. 53, no. 1-4, pp. 185-191, Jan. 2001.

[10] K. Kawahata, P. C. de Vries, Y. Nagayama, S. Inagaki, T. Tokuzawa, K. Tanaka, and S. Sasao, "The 14-channel grating polychromator for ECE measurements in LHD," Rev. Sci. Instrum., vol. 72, no. 1, pp. 387-390, Jan. 2001.

[11] K. Kawahata, Y. Nagayama, S. Inagaki, and Y. Ito, "Broadband electron cyclotron emission radiometry for the large helical device," Rev. Sci. Instrum., vol. 74, no. 3, pp. 1449-1452, Mar. 2003.

[12] Y. Nagayama, K. Kawahata, S. Inagaki, S. Kubo, K. Narihara, and N. Ohyabu, "Electron cyclotron emission diagnostics in the large helical device," J. Plasma Fusion Res., vol. 79, no. 6, pp. 601-607, June 2003.

[13] S. Preische, P. C. Efithimion, and S. M. Kaye, "Oblique electron cyclotron emission for electron distribution studies," Rev. Sci. Instrum., vol. 74, no. 3, pp. 1449-1452, Mar. 2003.

[14] S. V. Mirnov and I. B. Semenov, "Investigation of macroscopic instabilities of plama column in tokamak devices," in Proc. 4th Conf. Plasma Physics Controlled Nuclear Fusion Res., vol. 2. Vienna, Austria, 1971, pp. $401-406$.

[15] J. C. Hosea, C. Bobeldijk, and D. J. Grove, "Investigation of macroscopic instabilities of plama column in tokamak devices," in Proc. 4th Conf. Plasma Physics Controlled Nuclear Fusion Res, vol. 2. Vienna, Austria, 1971, pp. 425-440.

[16] K. Narihara et al., "Observation of the 'self-healing' of an error field island in the large helical device," Phys. Rev. Lett., vol. 87, p. 135002 , Sept. 2001.

[17] N. Ohyabu et al., "Island dynamics in the large-helical-device plasmas," Phys. Rev. Lett., vol. 88, p. 055005 , Feb. 2002.

[18] Y. Nagayama, K. Kawahata, S. Inagaki, B. J. Peterson, and S. Sakakibara, "Sawtooth oscillation in current-carrying plasma in the large helical device," Phys. Rev. Lett., vol. 90, p. 205 001, May 2003.

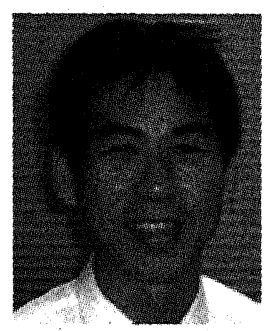

Yoshio Nagayama received the Ph.D. degree in plasma physics from the University of Tokyo, Tokyo, Japan, in 1979.

After working for plasma control in tokamak and RFP at the University of Tokyo, he was engaged in the imaging diagnostics or tomography and investigated the magnetohydrodynamic instabilities and the reconnection in TFTR tokamak at the Princeton Plasma Physics Laboratory, Princeton, NJ. Since 1994, he has been with the Large Helical Device Group, National Institute for Fusion Science, Toki, Japan, where he is currently investigating plasma physics for nuclear fusion, especially the electron cyclotron emission diagnostics and the data analysis system.

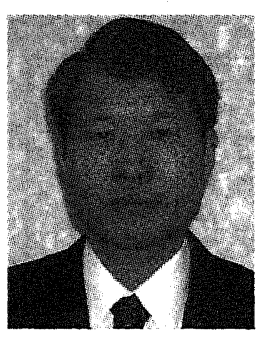

K. Kawahata received the Ph.D. degree in physics from Nagoya University, Nagoya, Japan, in 1976.

After receiving the Ph.D. degree, he joined the research staff at the Institute of Plasma Physics, Nagoya University, Nagoya, Japan, specializing in millimeter/submillimeter wave diagnostics for magnetic fusion plasmas. Since 1989, he has been with the Large Helical Device Group, National Institute for Fusion Science, Toki, Japan. His current research interests include the experimental physics of the Large Helical Device and the development of millimeter/submillimeter wave diagnostics needed to investigate plasma characteristics in fusion experiments.

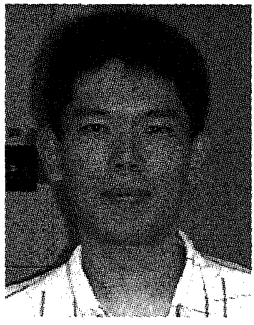

Shigeru Inagaki received the $\mathrm{Ph} . \mathrm{D}$. degree in heliac plasma confinement physics from Tohoku University, Sendai, Japan, in 1997.

He moved to the National Institute for Fusion Science, Toki, Japan, in 1997. He has been engaged in diagnostics, especially electron cyclotron emission measurements in LHD. He is currently investigating electron heat transport in LHD plasma.

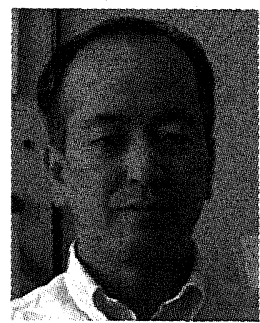

Tomohiro Morisaki received the M.S. and Ph.D. degrees from Kyushu University, Kyushu, Japan.

Since 1995, he has worked with the Large Helical Device Group, National Institute for Fusion Science, Toki, Japan. His current research interests include edge plasma physics and active edge control with divertor, together with the edge diagnostics.

K. Narihara photograph and biography not available at the time of publication. 